PoS $\quad \begin{aligned} & \text { PROCEEDINGS } \\ & \text { OF SCIENCE }\end{aligned}$

\title{
WG2 Highlights: Small-x, Diffraction and Vector Mesons
}

M. DEILE, L. GOERLICH, S. MUNIER

CERN, Geneva, E-mail: mario.deile@cern.ch

Institute of Nuclear Physics PAN, Cracow, E-mail: lidia.goerlich@ifj.edu.pl

Centre de physique théorique, École Polytechnique, CNRS, Palaiseau, E-mail:

stephane.Munierecpht.polytechnique.fr

XXII. International Workshop on Deep-Inelastic Scattering and Related Subjects

28 April - 2 May 2014

Warsaw, Poland 


\section{Theory}

In order to introduce the basic theoretical tools used in small-x physics, let us start with the example of the formulation of electroproduction of vector mesons. (An extensive review was given by Besse [1]). The standard theoretical description of this kind of process relies on the so-called dipole factorization, or alternatively the $k_{\perp}$-factorization of the scattering amplitude $\mathscr{M}$ (see Fig. 1). In both cases, the cross section is written as the convolution in the transverse plane of a function which characterizes the projectile, and another function which describes its interaction with the target.
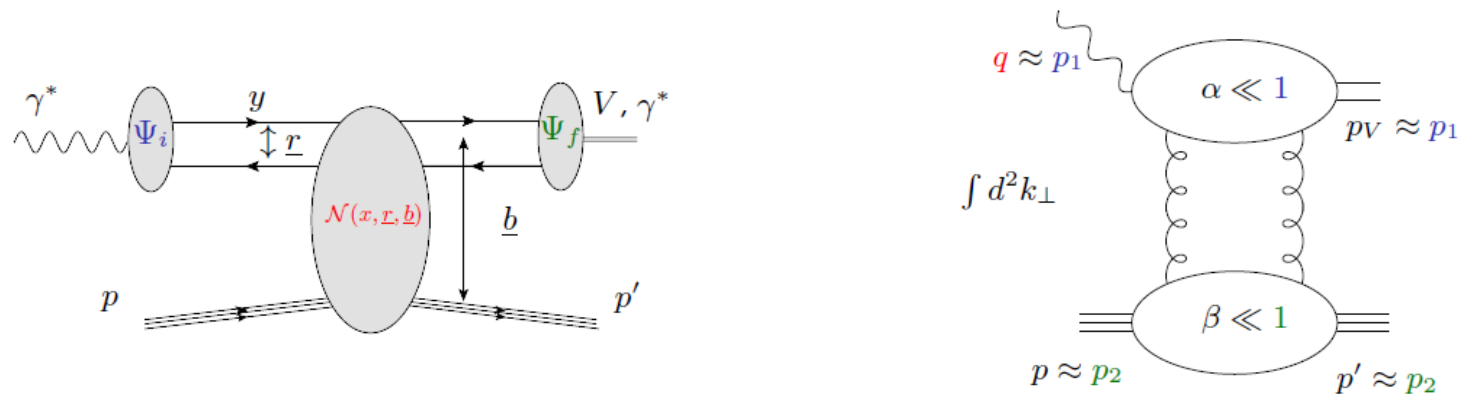

Figure 1: Schematic representation of dipole factorization (left) and $k_{\perp}$-factorization (right). (From Ref. [1]).

Dipole factorization reads, schematically

$$
\mathscr{M}_{\lambda_{V}, \lambda_{\gamma}}=i s \int \mathrm{dy} \int \mathrm{d}^{2} \underline{\mathrm{r}} \Psi_{\lambda_{\mathrm{V}}}^{*}(\mathrm{y}, \underline{\mathrm{r}}) \Psi_{\lambda_{\gamma}}(\mathrm{y}, \underline{\mathrm{r}}) \hat{\sigma}(\mathrm{x}, \underline{\mathrm{r}})
$$

where the $\Psi$ 's are the wave functions of the photon and of the meson respectively, $\hat{\sigma}$ is the dipole cross section depending on Bjorken- $x$ (when quantum evolution is included; at leading order, the dipole cross section is just given by two-gluon-exchange diagrams and has no $x$-dependence), and on the dipole size $\underline{r} . s$ is the squared center-of-mass energy.

The $k_{\perp}$-factorization formula instead reads

$$
\mathscr{M}_{\lambda_{V}, \lambda_{\gamma}}=i s \int \frac{\mathrm{d}^{2} \underline{\mathrm{k}}}{(2 \pi)^{2}\left(\underline{k}^{2}\right)^{2}} \Phi^{\gamma^{*}\left(\lambda_{\gamma}\right) \rightarrow V\left(\lambda_{V}\right)}(\underline{k}) \Phi^{P \rightarrow P}(-\underline{k})
$$

where $\underline{k}$ is the transverse momentum of the exchanged gluon and $\Phi$ the so-called impact factors. The proton impact factor is related to the unintegrated gluon distribution and the latter gets an $x$-dependence through quantum evolution.

Many other processes may be formulated in the dipole picture or with the help of $k_{\perp}$-factorization: Inclusive and diffractive cross sections, but also semi-exclusive processes in proton and nucleus scattering.

\subsection{Formal Developments at Small $x$}

The simple forms (1.1) and (1.2) assume a number of approximations, justified in the asymptotic limit of very high energies, namely for $x \rightarrow 0$. A highlight of the recent years has been the various attempts to go beyond these approximations. A review talk was given by Beuf [2].

Dipole factorization formulae are a priori valid only in the case of an infinitely thin target. Altinoluk reported on a systematic expansion of the gluon propagator in the background field of the 
target in powers of the size of the target in the case of inclusive gluon production in proton-nucleus scattering. Surprisingly but interestingly enough, the leading term in this expansion vanishes [3].

Leading-order quantum evolution is almost trivial to account for in the dipole formalism. It is enough to compute the eikonal emission of a single gluon in the wavefunction of the projectile, from which one obtains the so-called Balitsky-Kovchegov (BK) equation or Jalilian-Marian-IancuMcLerran-Weigert-Leonidov-Kovner (JIMWLK) equation, which promote the fixed-order calculation to its resummation to all orders in the coupling constant. The JIMWLK equation has been known at leading-logarithmic (LL) order for a long time. The current state-of-the-art is the nextto-leading- $\log (1 / x)$ (NLL) accuracy. A priori, about 30 different diagrams enter the calculation. Mulian presented a new derivation [4] of the NLL kernel of the JIMWLK equation, which makes use of clever tricks in order to avoid the calculation of all the latter diagrams.

Another way to go beyond leading order is to treat exactly the kinematics of the emission of the gluon in the wavefunction. Re-establishing the exact kinematics is empirically known to account for a large part of the subleading corrections. How to implement the kinematical constraint was known in momentum space: Beuf presented a way to enforce it in coordinate space [5].

The computation of the impact factor for the production of heavy quarks at next-to-leading order accuracy was also presented by Deak [6].

The dipole factorization formula should hold in the small- $x$ regime only. But according to Besse [1], it seems that for (at least) one particular helicity amplitude in the electroproduction of vector mesons, the equations valid at large- $x$ exhibit the factorization of the overlap of the wavefunctions, much as in the dipole model picture.

Systematic analytical methods are known in QCD only in the regime in which the coupling is small. However, one may try and extrapolate the collinearly-improved Balitsky-Fadin-KuraevLipatov (BFKL) equation supplemented with the kinematical constraint to the strong-coupling regime. Kutak derived a possible extension of the BK equation at strong coupling, and obtained in particular a conjecture for the rapidity-dependence of the saturation scale [7]. Another popular approach to the strong-coupling regime is through the AdS/CFT correspondence: Djurić et al. [8] managed to get a good description of vector-meson production at HERA from such a calculation, but at this stage, many phenomenological parameters still need to be determined from fits to the data.

\subsection{Theory versus Experiments: Phenomenology from HERA to LHC}

\subsubsection{Dipole Models}

In the case of dipole models, making contact with the HERA data for deep-inelastic scattering requires a model for the dipole-proton scattering amplitude. There are essentially two classes of models (see Fig. 2): the so-called IP-sat model, which essentially consists in an exponentiation of the DGLAP-evolved gluon density, and the b-CGC model (CGC stands for "colour glass condensate") which is closer to the solution to the BK/JIMWLK equations. Rezaeian presented a comparison of the predictions of the two classes of models [10]: They differ only at large momentum transfer and at very small $x$, and give generally a good description of all HERA data, including the combined data. Exclusive diffractive photoproduction of $J / \psi$ in proton-proton collisions can be 

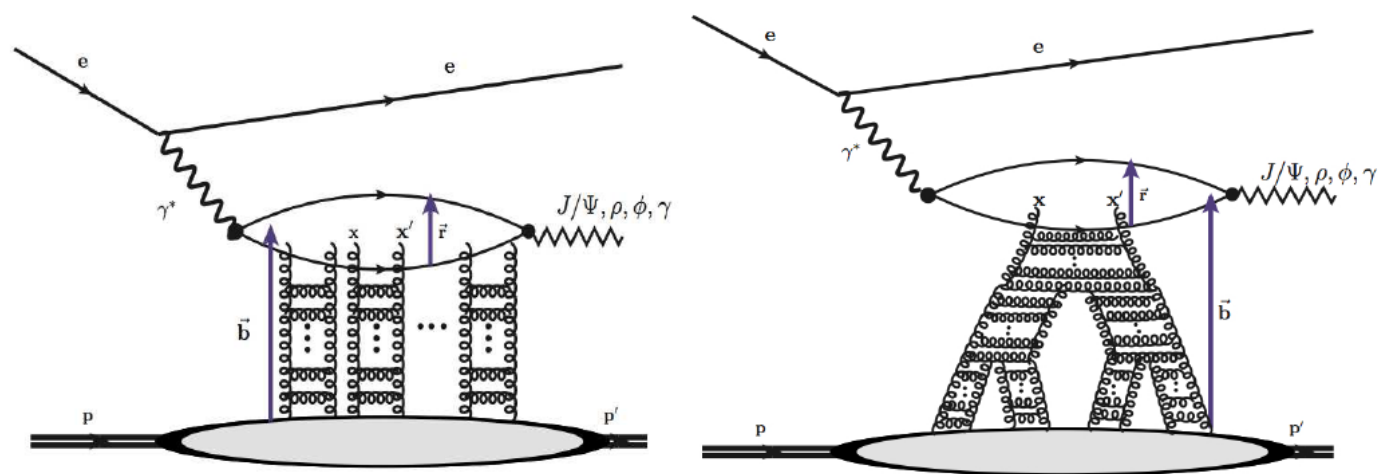

Figure 2: Two classes of dipole models: IP-sat (left) and b-CGC (right). In each case is represented a generic diagram contributing to the electroproduction of vector mesons. (From Ref. [9]).

described in the very same model: It seems that the recent LHCb data is in favor of CGC/saturation models.

Kowalski presented an update of the fits of the IP-sat model using the newest HERA data [11]. An interesting point is that the fits in the small- $x$ region are now sensitive to the valence quarks, due to the very high accuracy of the data.

The model which represents most fully the actual QCD dynamics may be the one presented by Mantysaari, where the dipole cross section is the numerical solution of the BK evolution with running coupling, and with an initial condition given by the McLerran-Venugopalan (MV) model [12]. It was shown that the LHC data for single hadron inclusive production called for a modification of the MV model. Mantysaari also showed that saturation effects are clearly seen in diffractive photoproduction of $J / \psi$ in the ultraperipheral collision of nuclei, already for values of $x$ of the order of $10^{-3}$ [13] (see Fig. 3).

\subsection{2 $\mathrm{k}_{\perp}$-factorization}

A variety of small- $x$ processes can be formulated with the help of $k_{\perp}$-factorization, and compared to the LHC data: Mueller-Navelet jets, forward (di-,tri-)jets, photo or hadroproduction of $J / \psi$, Higgs production as well as exclusive muon pairs have been discussed at this meeting.

The measurement of the cross section for the inclusive production of forward jets of similar transverse momenta but separated by a large rapidity interval, the so-called Mueller-Navelet jets, was advertised as being one of the best tests of $k_{\perp}$-factorization and of BFKL dynamics at hadron colliders. Nowadays, one also deems the distribution of the azimuthal angle between the jets a good probe of QCD in the small- $x$ regime, which a priori predicts a significant decorrelation. However, the status of this theory/experiment confrontation has been quite confusing at the LHC: Indeed, a first calculation using the BFKL formalism at next-to-leading order [14] seemed to completely fail to describe the azimuthal correlations. However, the new calculation presented by Ducloue at this workshop shows a perfect agreement. The difference is in the choice of the renormalization scale: In the former calculation, it was fixed, while in the latter, it comes out of the Brodsky-LepageMackenzie (BLM) scale-fixing procedure [15].

Maciula presented an interesting process which could compete with the usual (BFKL) MuellerNavelet jets [16]. It is based on the observation that there may be two simultaneous hard partonparton scatterings in one proton-proton interaction, giving rise to two jets in each forward rapidity 


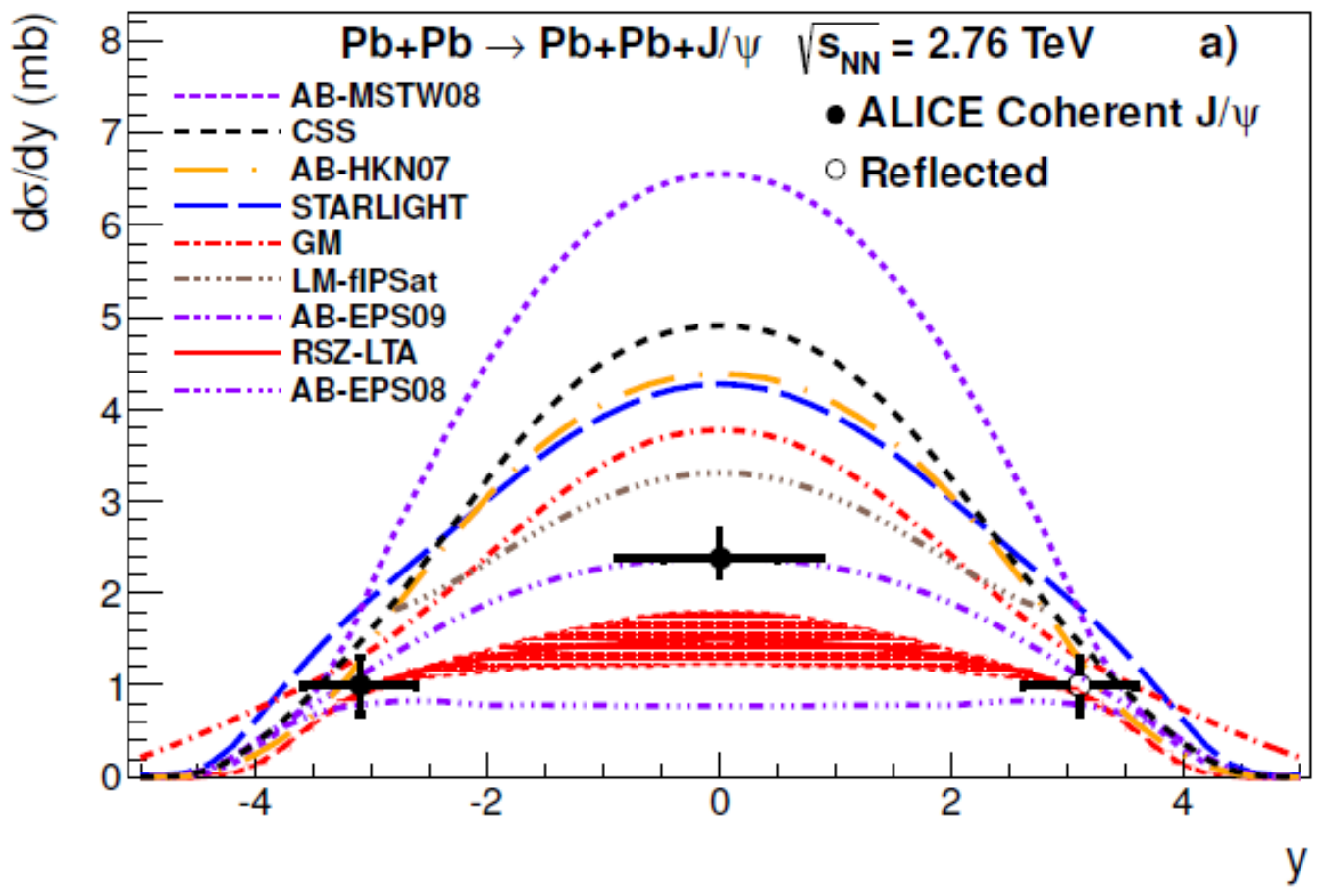

Figure 3: Diffractive photoproduction of $J / \psi$ in ultraperipheral lead-lead collisions, compared to different models. The one that fits best is a saturation model while unshadowed models (such as AB-MSTW08) clearly fails.

region; A subset of two of those could be tagged as Mueller-Navelet jets. Since the two partonic subprocesses are not correlated, the angular dependence is flat. The cross section for these doubleparton scattering events becomes significant at high rapidity.

Forward jets in the same rapidity region may be used as a probe of saturation effects, as shown by Kotko. In this case, the relevant factorization formula is hybrid, mixing $k_{\perp}$-factorization on one side and collinear factorization on the other side. The differential cross section for the production of a given set of particles $X$ in the collision of two hadrons $A$ and $B$ reads

$$
\mathrm{d} \sigma_{\mathrm{AB} \rightarrow \mathrm{X}}=\int \frac{\mathrm{d}^{2} \mathrm{k}_{\perp \mathrm{A}}}{\pi} \int \frac{\mathrm{dx}_{\mathrm{A}}}{\mathrm{x}_{\mathrm{A}}} \int \mathrm{dx}_{\mathrm{B}} \mathscr{F}\left(\mathrm{x}_{\mathrm{A}}, \mathrm{k}_{\perp \mathrm{A}}, \mu\right) \mathrm{f}_{\mathrm{b} / \mathrm{B}}\left(\mathrm{x}_{\mathrm{B}}, \mu\right) \mathrm{d} \sigma_{\mathrm{g}^{*} \mathrm{~b} \rightarrow \mathrm{X}}\left(\mathrm{x}_{\mathrm{A}}, \mathrm{x}_{\mathrm{B}}, \mathrm{k}_{\perp \mathrm{A}}, \mu\right) .
$$

$\mathscr{F}$ is the unintegrated gluon distribution in the colliding object $A, f_{b / B}$ the integrated parton density of type $b$ (quarks or gluons) in the object $B$, and $\mathrm{d} \sigma$ is the partonic sub-cross section. The variables are the standard ones for these functions. A wealth of observables may be formulated with the help of such a factorization formula. With dijets, useful observables are the azimuthal decorrelation and the transverse momentum spectrum [17]. But trijet observables may also be formulated, as well as forward-central dijet correlations, and so on.

Cisek explained in particular that exclusive photoproduction of $J / \psi$ mesons in $e p$ and $p p$ collisions turns out to be very sensitive to the form of the quarkonium wave function [18]. Such processes are also good tests for unintegrated gluon distribution functions.

As for hadroproduction of $J / \psi$ mesons instead, one needs to take into account multiple-gluon exchanges. Motyka indeed observed that colour singlet rescattering corrections are sizeable: At 
Tevatron and LHC, they are larger than standard colour singlet contributions and may make up to $25 \%$ of direct $J / \psi$ cross section at moderate values of the transverse momentum [19].

As for hadroproduction of the Higgs boson, Szczurek showed that this process is particularly sensitive to the form of the unintegrated gluon distribution functions. Next-to-leading order corrections need to be taken into account, as well as electroweak corrections [20].

Finally, Schaefer showed that the central exclusive production of lepton pairs in proton-proton collisions with large transverse momenta receives a large contribution from proton dissociation events [21].

\section{Experiments at HERA}

For more than 20 years diffractive processes have been extensively studied at the electronproton colider HERA by the H1 and ZEUS Collaborations and the main results of these investigations were reviewed at DIS 2014 [22]. In this section we focus on recent measurements.

In the framework of a QCD collinear factorization the cross section for the diffractive DIS (DDIS) process factorizes into universal diffractive parton distribution functions (DPDFs) and hard subprocess cross sections, calculable within perturbative QCD. The DPDFs extracted from inclusive measurements have been applied as input to QCD calculations of many diffractive processes and this approach have been successful in the prediction of hard diffractive production in DIS. However, the factorization breaking was observed for hard processes in diffractive hadron-hadron scattering when DPDFs extracted from HERA data were applied, for example, to diffractive $p \bar{p}$ scattering at the Tevatron. This factorization breaking can be explained in terms of multiple scattering effects, which occur in the presence of beam remnants and lead to the destruction of the rapidity gaps associated with the diffractive process. Rapidity gap survival effects can be investigated at HERA in hard diffractive dijet photoproduction.

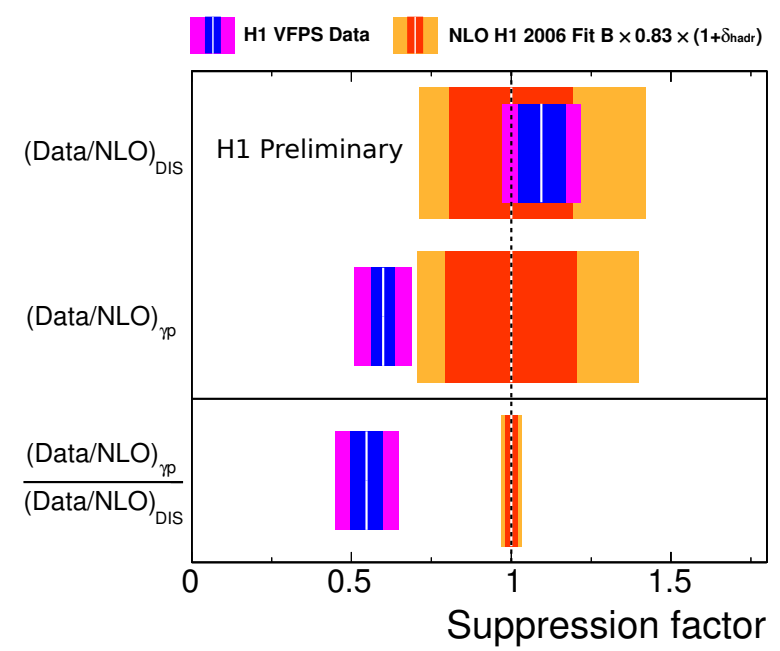

Figure 4: Diffractive dijet DIS and photoproduction integrated cross sections normalised to NLO QCD calculations and double ratio of data to NLO QCD prediction for photoproduction and DIS.

New measurements of proton-tagged diffractive dijets in photoproduction $\left(Q^{2}<2 \mathrm{GeV}^{2}\right)$ and DIS $\left(4 \mathrm{GeV}^{2}<\mathrm{Q}^{2}<80 \mathrm{GeV}^{2}\right)$ have been reported by $\mathrm{H} 1$ [23]. The cross sections for diffractive dijet production in both processes are compared to NLO QCD calculations based on DPDFs. To 
test reliably the collinear QCD factorization the double ratio of measured to predicted cross sections in photoproduction to the corresponding ratio in DIS was determined (Fig. 4). Clear evidence for QCD factorization breaking in diffractive dijet photoproduction with respect to the same process in DDIS is observed and the suppression factor is equal to $0.55 \pm 0.10$ (data) +0.02 (theor.). This result confirms previous H1 measurements based on large rapidity gap method. However, ZEUS do not observe factorization breaking in diffractive photoproduced jets.

The exclusive production of dijets in DDIS has been measured by ZEUS for the first time at HERA [24]. The shape of the jet azimuthal angle distribution measured in the virtual photon pomeron rest frame is sensitive to the production mechanism of diffractive exclusive dijets. As shown in Fig. 5 (left) the data favour fully perturbative calculations of the two-gluon exchange model over the boson-gluon fusion (BGF) mechanism, in which a partonic structure of the pomeron described by DPDFs is assumed.
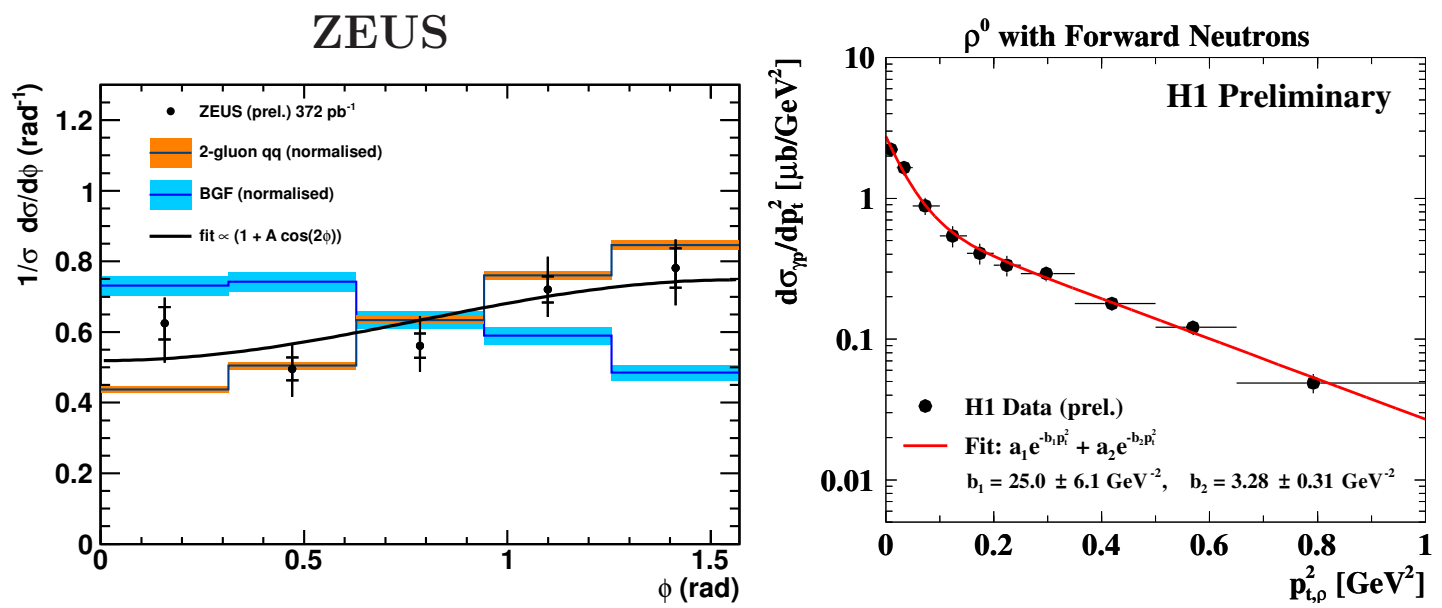

Figure 5: The normalised cross section $1 / \sigma \mathrm{d} \sigma / \mathrm{d} \phi$ for the exclusive production of dijets in DDIS as a function of the azimuthal angle $\phi$ between the plane spanned by the incoming and scattered lepton momenta and the plane defined by the exchanged photon and dijet momenta in the virtual photon-pomeron rest frame (left, [24]). Cross section $\mathrm{d} \sigma_{\gamma_{\mathrm{p}}} / \mathrm{dp}_{\mathrm{t}}^{2}$ as a function of $p_{t}^{2}$ of $\rho$ mesons produced in the reaction $\gamma^{*} p \rightarrow \rho^{\circ} \pi^{+} n$ (right, [25]).

H1 has measured exclusive photoproduction of $\rho^{\circ}$ mesons associated with leading neutrons, $\gamma^{*} p \rightarrow \rho^{\circ} \pi^{+} n$, with the aim to extract for the first time experimentally the elastic $\gamma \pi$ cross section [25]. The data are interpreted in terms of the double peripheral process (DPP), involving $\pi$-exchange at the proton vertex followed by elastic scattering of this pion on the photon from the electron beam, $\gamma \pi^{+} \rightarrow \rho^{\circ} \pi^{+}$. This interpretation is confirmed by the behaviour of the differential cross section $\mathrm{d} \sigma_{\gamma p} / \mathrm{dp}_{\mathrm{t}}^{2}$ of $\rho$ mesons shown in Fig. 5. The $p_{t}^{2}$ distribution is fitted by two exponential functions with distinctly different slopes as expected for DPP reactions. The elastic photon-pion cross section extracted in the one-pion-exchange approximation at average energy of the photon-pion system $\left\langle W_{\gamma \pi}\right\rangle=22 \mathrm{GeV}$ is $\sigma\left(\gamma \pi^{+} \rightarrow \rho^{\circ} \pi^{+}\right)=\left(2.03 \pm 0.34_{\exp } \pm 0.51_{\text {model }}\right) \mu \mathrm{b}$.

\section{Experiments at Hadron Colliders}

\subsection{Elastic Scattering}

A new elastic scattering measurement has been performed by TOTEM [27]. For the first time 
at the LHC, the kinematic acceptance was extended into the domain of very low squared fourmomenta, $|t| \geq 6 \times 10^{-4} \mathrm{GeV}^{2}$, where the effects of the Coulomb-nuclear interference manifest themselves (Fig. 6), providing information on the phase of the hadronic scattering amplitude. The analysis is still in progress.

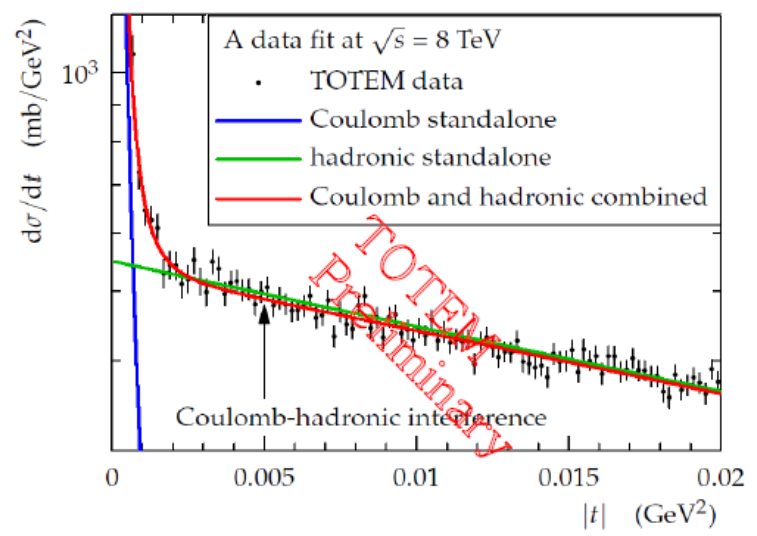

Figure 6: Distribution of $|t|$ in pp elastic scattering at $\sqrt{s}=8 \mathrm{TeV}$.

\subsection{Single and Double Diffractive Dissociation}

Recent results on Single Diffraction (SD) at LHC have been reported by CMS [26] and TOTEM [27]. While CMS tags the events by rapidity gap and reconstructs them with information from the central tracker, TOTEM detects the single surviving proton with the Roman Pots (RPs) and reconstructs the diffractive mass based on the rapidity gap in the forward trackers T1 and T2. For three ranges of diffractive mass, TOTEM has fitted the differential cross-section $\mathrm{d} \sigma / \mathrm{dt}=\mathrm{A} \exp -\mathrm{B}|\mathrm{t}|$ and determined the exponential slope $B$ which was found to decrease with increasing mass. Within their respective acceptances, CMS and TOTEM obtain integrated SD crosssections of $\sigma_{\mathrm{CMS}}^{\mathrm{SD}}=(4.27 \pm 0.04 \text { (stat })_{-0.58}^{+0.65}$ (syst) $) \mathrm{mb}$ for $12.6 \mathrm{GeV}<M<400 \mathrm{GeV}$, and $\sigma_{\mathrm{TOTEM}}^{\mathrm{SD}}=$ $(6.5 \pm 1.3) \mathrm{mb}$ for $3.4 \mathrm{GeV}<M<1100 \mathrm{GeV}$. The comparison with measurements by other experiments at different energies is shown in Fig. 7 (left) where the CMS value has been corrected for the invisible low mass events by K. Goulianos [28]. The TOTEM value is shown without such a correction, but adding their indirect low-mass measurement, $\sigma_{\text {inel, }} \mathrm{M}<3.4 \mathrm{GeV}=(2.62 \pm 2.17) \mathrm{mb}$, yields a result at a similar level.
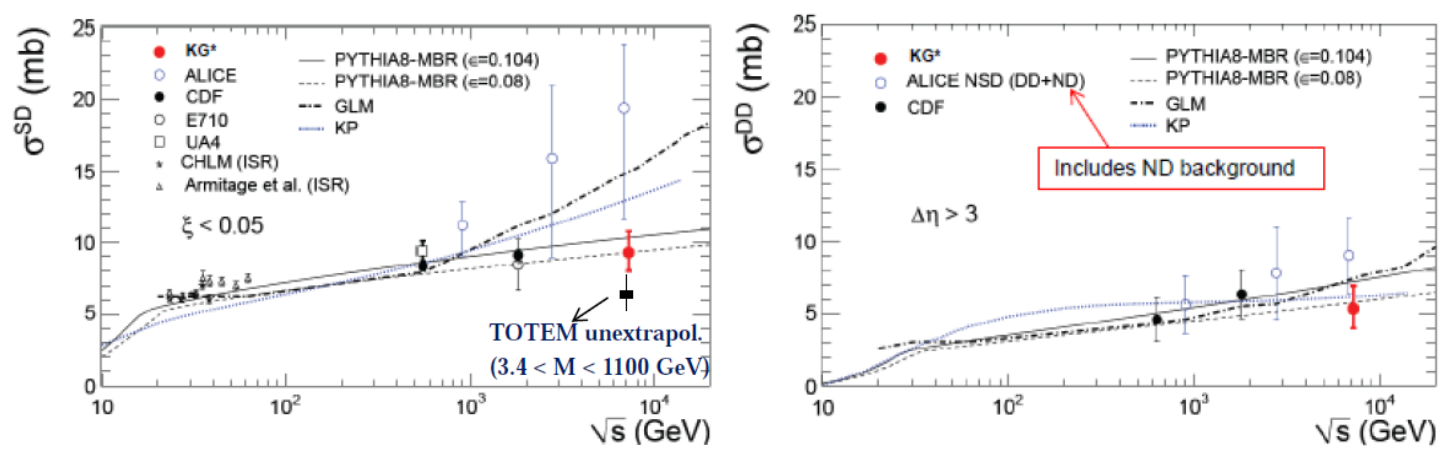

Figure 7: Compilation of SD (left) and DD (right) cross-section measurements and model predictions [28]. The TOTEM point in SD has been added by the conveners. 


\subsection{Exclusive Production Via Colourless Exchanges}

The mechanism of central exclusive production offers a very clean event signature with kinematic redundancy between the central system and the surviving beam particles (leading protons or ions). Depending on the exchange mechanism of the process considered (i.e. any combination of pomeron, photon and possibly odderon), different selection rules for the spin-parity numbers of the central state apply. Hence, the angular distribution of a given observed resonance can be used for the spin-parity analysis of that state. In the search for new particles, well established states with known quantum numbers can serve as "standard candles". For example, the Durham group's predictions for central exclusive production can be benchmarked against LHCb's observation of the $\chi_{c 0.1 .2}$ mesons [29]. Particularly interesting discovery potential lies in possible glueball states whose production is expected to be favoured by pomeron-pomeron exchanges.

Theorist's interest in future central production experiments is also nourished by the hope to shed some light on the spin of the pomeron: while present data are compatible both with vector and tensor pomerons, theory prefers the tensor option [30].

Present experiments show a high level of activity in central production studies. The following paragraphs give some examples presented at DIS 2014.

The STAR experiment at RHIC [31] has profitted from their two-arm Roman Pot spectrometer to tag both surviving protons of central diffractive processes. The mass spectrum of exclusive events with a two-pion final state at $\sqrt{s}=200 \mathrm{GeV}$ in pp collisions is shown in Fig. 8. It exhibits a resonance structure very similar to the one observed at $\sqrt{s}=63 \mathrm{GeV}$ in the Axial Field Spectrometer experiment at the CERN ISR [32]: both distributions show a distinctive drop at the $\mathrm{f}_{0}(980)$ resonance. To resolve the structures at higher masses, STAR is looking forward to new data in 2015 with 30-40 times more statistics. Similar studies - not presented at DIS 2014 - in the two-pion channel are being pursued by CDF, ALICE and CMS+TOTEM.
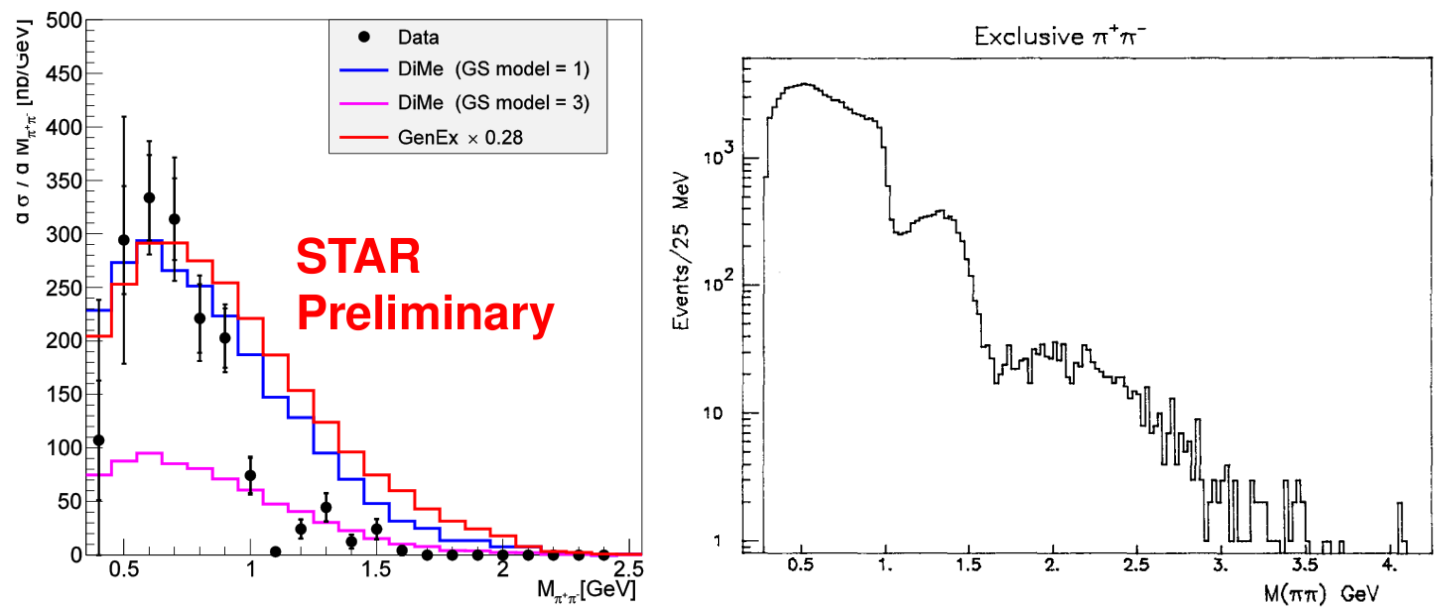

Figure 8: Mass distribution of central exclusive $\pi^{+} \pi^{-}$production at STAR (RHIC, $\sqrt{s}=200 \mathrm{GeV}$, left panel) and AFS (ISR, $\sqrt{s}=63 \mathrm{GeV}$, right panel).

CMS has studied the central production of $\mathrm{W}$ pairs via two-photon exchange. The observed cross-section is consistent with the standard model prediction and thus imposes new limits on anomalous quartic gauge couplings [26]. 
LHCb has measured a wealth of central exclusive processes with two muons in the final state. After studying the production of the $\chi_{c 0,1,2}$ states via pomeron-pomeron exchange (not presented at DIS 2014), they passed on to $J / \Psi$ and $\Psi(2 S)$ photoproduction (photon-pomeron exchange) [33] ${ }^{1}$. From the measured differential production cross-section, $\mathrm{d} \sigma(\mathrm{pp} \rightarrow \mathrm{pJ} / \Psi \mathrm{p}) / \mathrm{dy}$ (where $y$ is the rapidity), and the photon flux obtained via the Weizsäcker-Williams approximation, the photoproduction subprocess cross-section, $\sigma(\gamma \mathrm{p} \rightarrow \mathrm{J} / \Psi \mathrm{p})(W)$ at the photon-proton system mass $W$, was extracted and compared with measurements at HERA (Fig. 9, left). The LHCb points are found to be in marginal agreement with the $\mathrm{H} 1$ power-law fit, showing some deviations at large $W$. In addition to higher order effects being capable of explaining the deviation from a pure power-law behaviour, saturation effects may be important.

A similar study was carried out by ALICE [34] based on $J / \Psi$ photoproduction data in proton$\mathrm{Pb}$ collisions (Fig. 9, right). ALICE performed their own power-law fit $\sigma(\gamma \mathrm{p} \rightarrow \mathrm{J} / \Psi \mathrm{p})(W) \propto W^{\delta}$ and obtained an exponent $\delta$ in good agreement with $\mathrm{H} 1$ and Zeus.
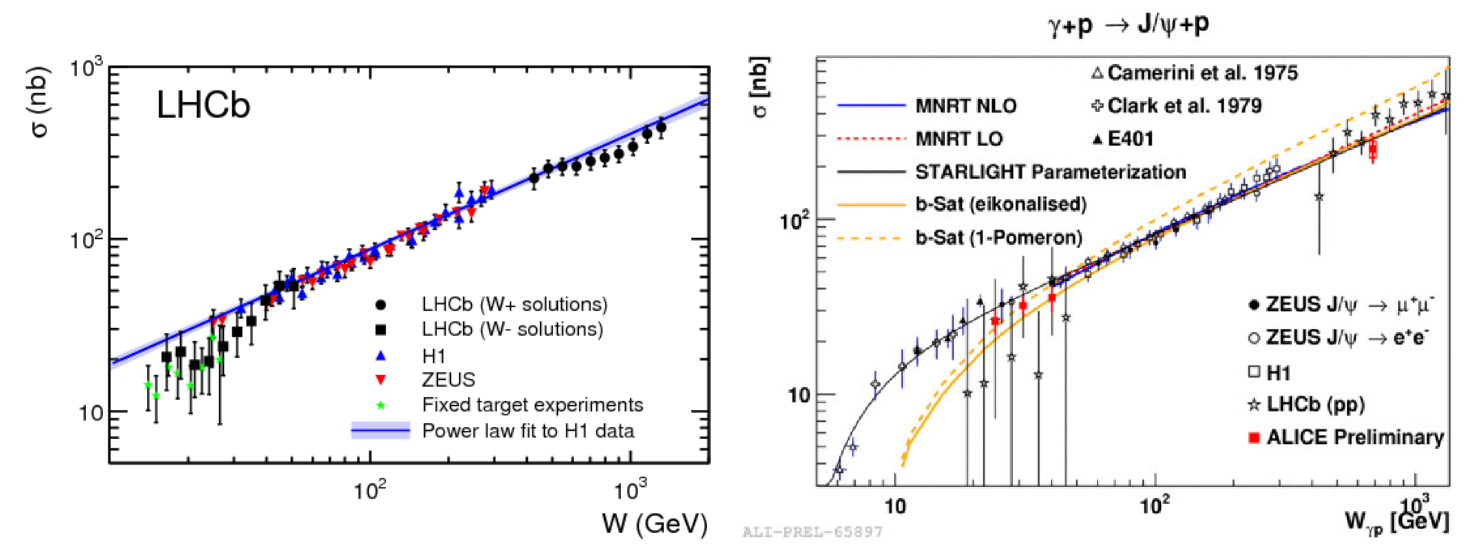

Figure 9: Photoproduction subprocess cross-section as a function of the proton-photon system mass. Left: $\mathrm{LHCb}$ measurement in pp collisions; right: ALICE measurement in $\mathrm{pPb}$ collisions. In both panels the HERA results are shown for comparison. In the right panel, the LHCb comparison points reflect an earlier measurement superseded by the left plot.

ALICE also performed extensive measurements of $J / \Psi$ photoproduction in ultraperipheral $\mathrm{Pb}-\mathrm{Pb}$ collisions [34]. The analysis has been carried out for central $(|y|<0.9)$ and forward $(2.6<$ $|y|<3.6)$ production rapidities, and distinguishing coherent and incoherent production via the transverse momentum of the detected di-muon decay system. The differential production crosssection, $\mathrm{d} \sigma /$ dy gives information on gluon shadowing in nuclei at low $x$ (see also Section 1.2.2, Fig. 3). Models incorporating this effect provide the best description of the data.

\subsection{Inclusive Particle Production}

\subsubsection{Pseudorapidity Density}

The CMS and TOTEM collaborations have published a common measurement of the charged particle pseudorapidity density distribution, $\mathrm{dN}_{\mathrm{ch}} / \mathrm{d} \eta$, over an $\eta$-range from 0 to 6.5 with a gap from 2.2 to 5.3 [26]. This measurement probes hadronisation processes and thus allows to constrain theoretical models that are also interesting for cosmic-ray simulations. The analysis was carried out

\footnotetext{
${ }^{1}$ See also Section 3.4.2 for non-exclusive quarkonium production.
} 
for three cases: an inclusive pp event sample (Fig. 10, left plot), a single-diffraction-enhanced sample (right plot, showing a much lower multiplicity), and a non-single-diffraction-enhanced sample (not shown here). The fact that no Monte Carlo generator describes the full $\eta$-range satisfactorily underlines the importance of the forward measurement.
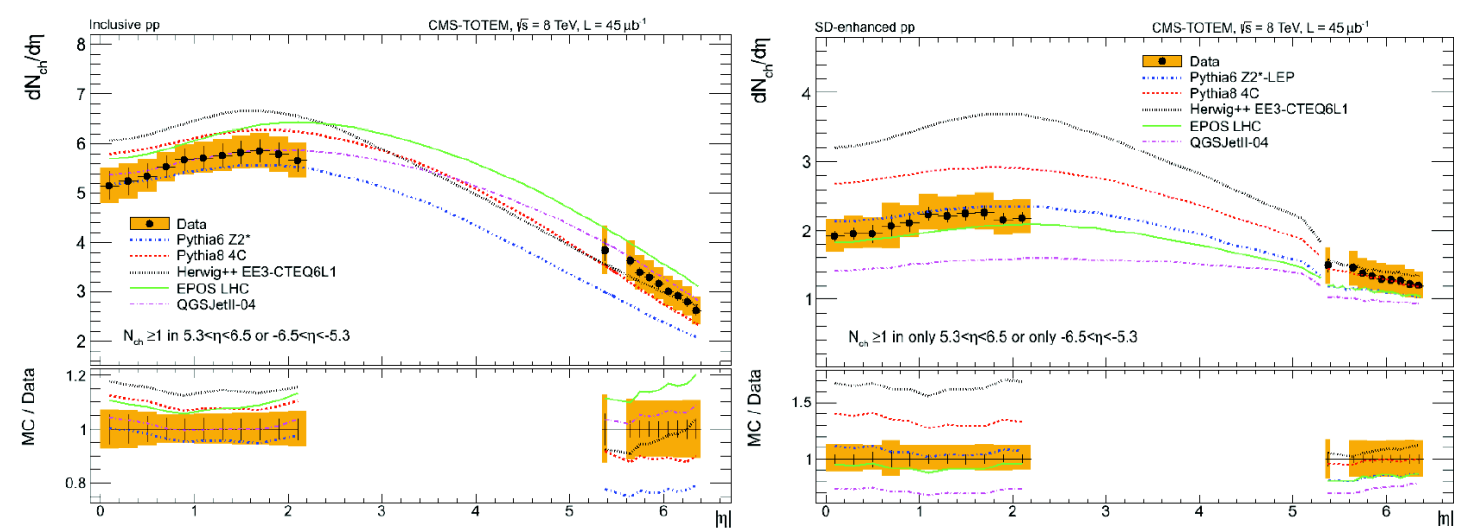

Figure 10: Charged particle pseudorapidity density distribution $\mathrm{dN} / \mathrm{d} \eta$ measured by CMS and TOTEM with a common data set triggered by the TOTEM forward tracker T2 with acceptance for more than $90 \%$ of all inelastic events. Left: inclusive data sample; right: data sample enriched in single diffractive events.

\subsubsection{Quarkonium Production}

Two presentations were dedicated to inclusive production of quarkonia. Figure 11 shows the differential production cross-section in rapidity for $\Phi(1020)$ (left, [35]), a mixture of šs/ūu/d̄d, and for different $\bar{c} c$ and $\bar{b} b$ states (right, [36]). For $J / \Psi$ a polarisation measurement was reported, yielding a small non-zero longitudinal value.
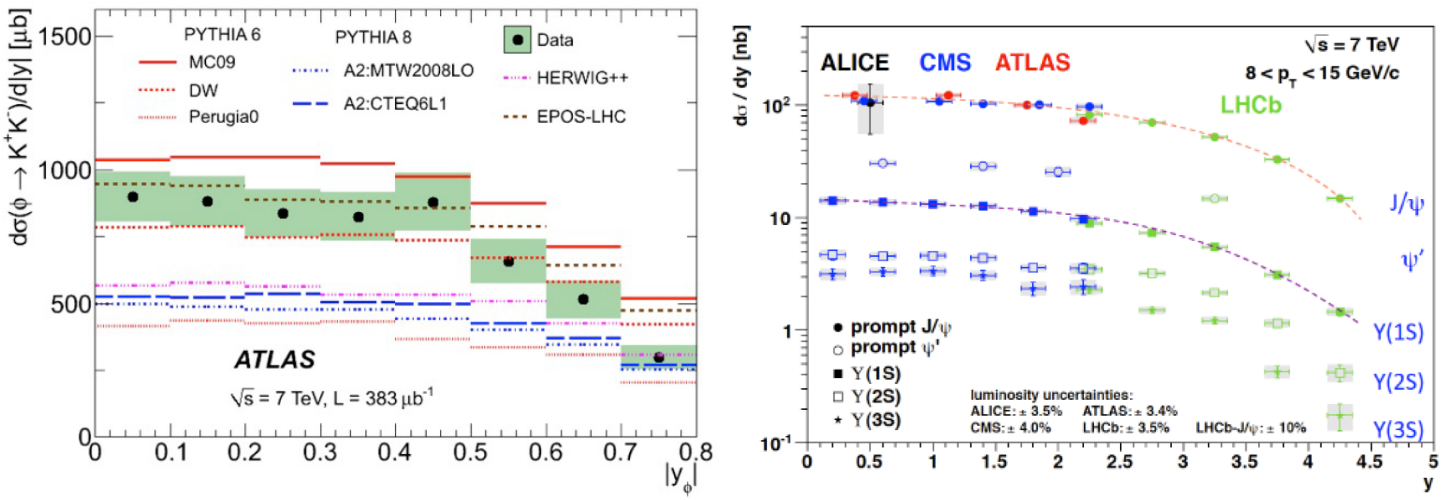

Figure 11: Quarkonium production cross-sections for $\Phi(1020)$ (left, [35]) and various $\bar{c} c$ and $\bar{b} b$ states (right, [36])

LHCb has also measured $J / \Psi$ production in p-Pb collisions [37]. The comparison with the p-p case enables the determination of the nuclear modification factor, probing Cold Nuclear Matter effects. The measurement shows the expected suppression of heavy quarkonia at large rapidities in $\mathrm{p}-\mathrm{Pb}$ collisions (Fig. 12). This effect is more pronounced for prompt production than for $J / \Psi$ from b-hadron decays. 

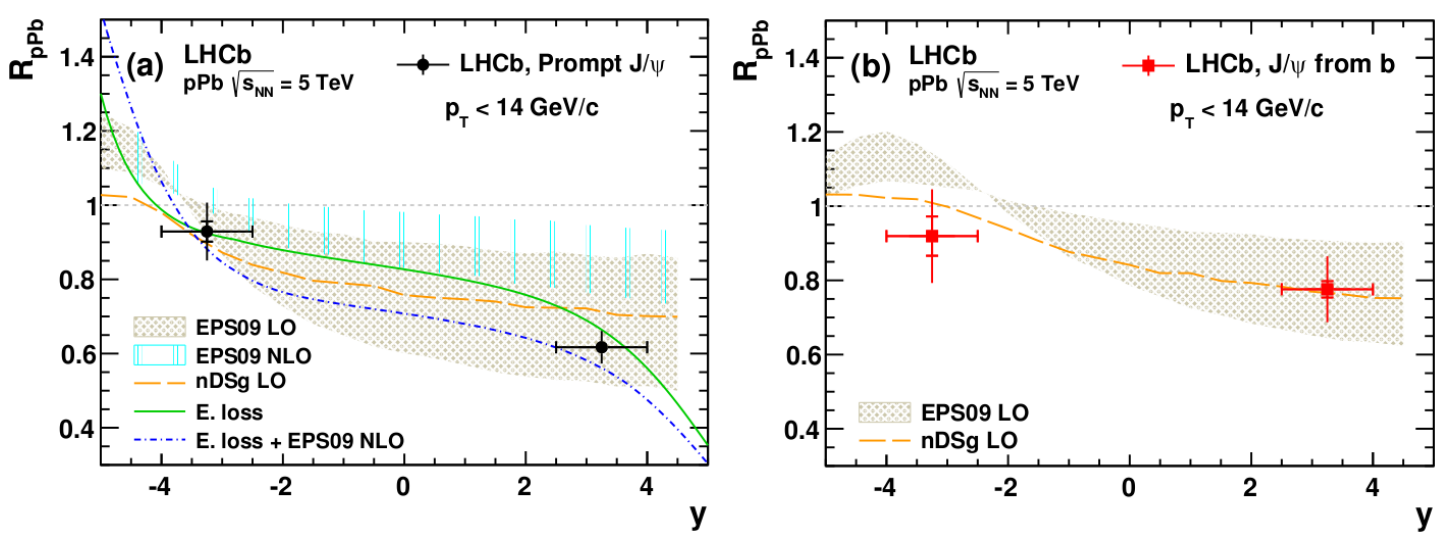

Figure 12: Nuclear modification factor $R_{\mathrm{pA}}=\frac{1}{N_{\mathrm{coll}}} \frac{\mathrm{d} \sigma_{\mathrm{pA}} / \mathrm{dy}}{\mathrm{d} \sigma_{\mathrm{pp}} / \mathrm{dy}}$ for $J / \Psi$ production in $\mathrm{p}-\mathrm{Pb}$ collisions; left: prompt production; right: via b-hadron decay.

\subsection{Probing Low-x QCD Dynamics with Jets}

Jet production in proton-proton collisions at the LHC is a valuable tool for investigating QCD dynamics at low $x$. Measurements of low transverse momentum jets produced in the forward region (at large rapidity $y$ ) are considered to be especially sensitive to BFKL effects. CMS has presented the combined differential inclusive jet cross sections, measured in the rapidity ranges $|y|<4.7$ and at low and high $p_{\mathrm{T}}$, using data collected with low and high pileup runs at $\sqrt{s}=8 \mathrm{TeV}$ (Fig. 13) [38]. The data are well described in a wide range of $p_{\mathrm{T}}$ and $y$ by DGLAP-based calculations including NLO corrections.

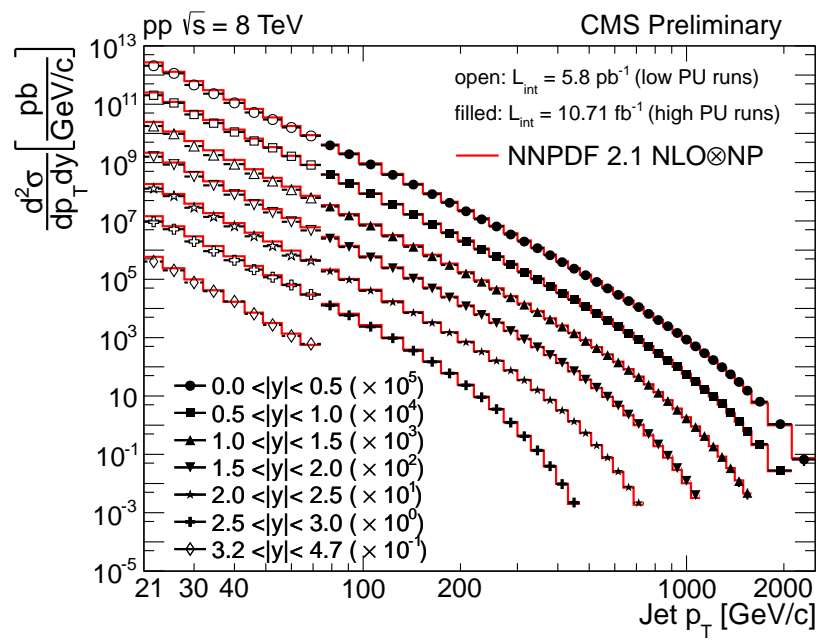

Figure 13: The combined differential inclusive jet cross sections as a function of $p_{\mathrm{T}}$ in different intervals of $y$ compared to NLO QCD predictions.

CMS has also discussed new results on the azimuthal correlation between forward and central jets at $\sqrt{s}=7 \mathrm{TeV}$ [39]. Events with at least one forward jet $(3.2<\eta<4.7)$ and one central jet $(|\eta|<2.8)$ with $p_{\mathrm{T}}>35 \mathrm{GeV}$ are selected and the azimuthal angle difference $\Delta \phi$ between the jets is tested for different separations in pseudorapidity, with the largest separation being $\Delta \eta=7.5$ units. To further investigate details of the parton radiation along the parton ladder the measurement is also performed for two subsamples, one where an additional jet with $p_{\mathrm{T}}>20 \mathrm{GeV}$ is required between the forward and the central jet (inside-jet tag scenario) and one with this inter-leading 
jet activity vetoed (inside-jet veto scenario). As shown in Fig. 14 for the inclusive dijet sample the correlation between jets decreases with increasing $\Delta \eta$. This tendency, also observed in the inside-jet tag scenario (not shown), is due to the fact that at large $\Delta \eta$ there is more phase space for the additional parton radiation. Fig. 15 shows a comparison of the azimuthal forward-central jet correlations for different jet topologies.
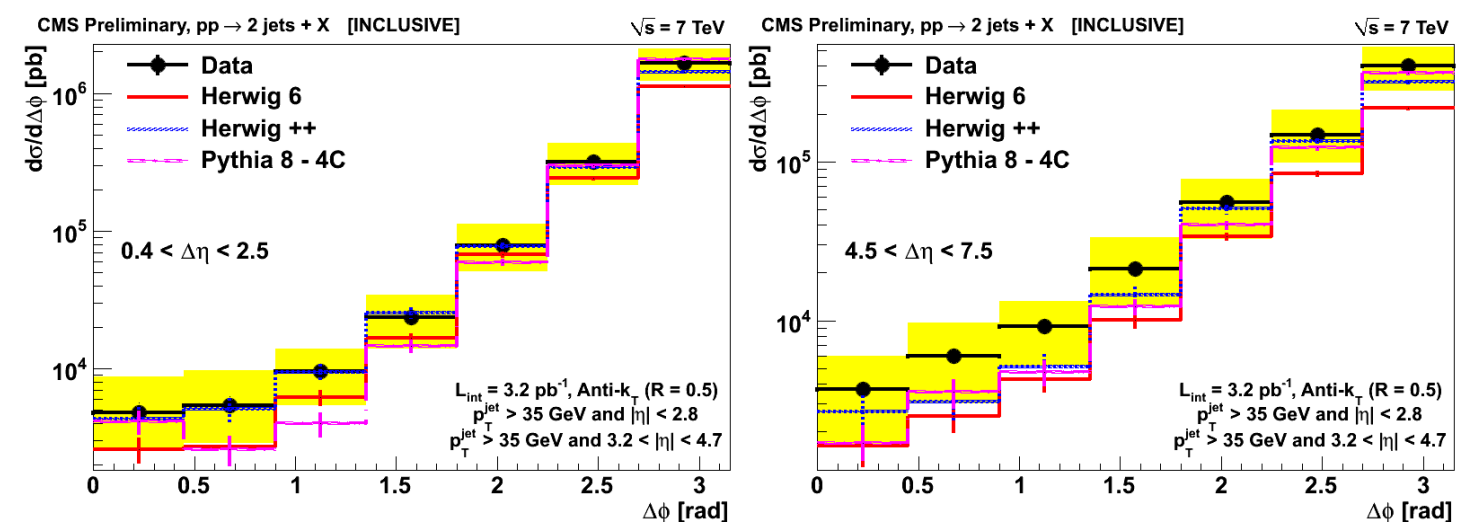

Figure 14: Differential forward-central dijet cross section as a function of $\Delta \phi$ in two intervals of $\Delta \eta$ compared with the predictions of DGLAP-based models.

In general the DGLAP-based generators (Herwig 6, Herwig ++ and Pythia 8-4C) describe well the measured observables. Herwig++ using angular ordered parton showers and cluster fragmentation in the hadronisation step as well as including multiple parton interactions as a part of the underlying event modelling, provides the best predictions describing the measured cross sections in shape and normalisation. In summary, the presented measurements of the azimuthal forwardcentral jet correlations show that the BFKL effects are not dominant in the available phase space region at the LHC.

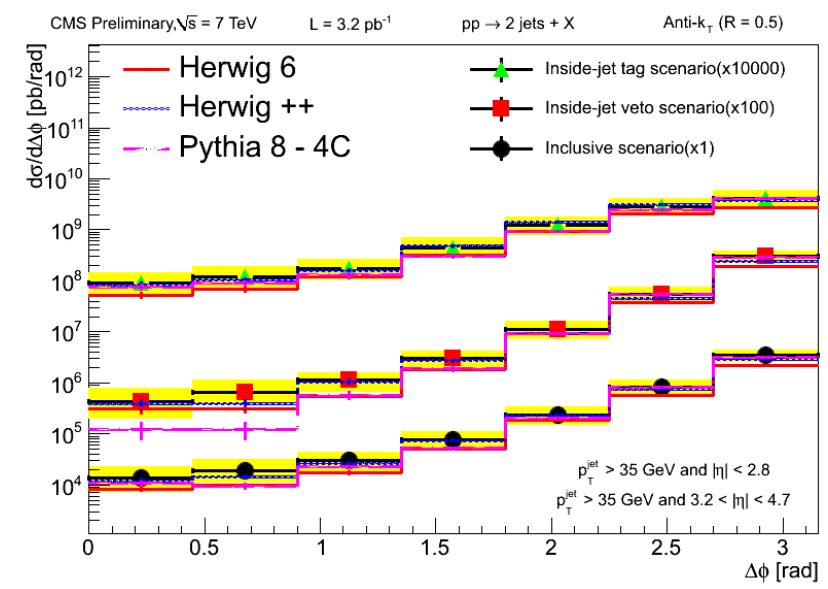

Figure 15: Differential forward-central dijet cross section as a function of $\Delta \phi$ for different jet topologies.

Similar conclusions on the absence of BFKL effects in experimental data have also been drawn from recent CMS measurements of the azimuthal angle decorrelations of Mueller-Navelet jets and from a study of ratios of the inclusive to exclusive dijet cross sections as a function of the rapidity separation between the two jets. Both topics were reviewed at DIS 2014 [38]. However, one has to note that recent NLL BFKL calculations of the azimuthal decorrelations of Mueller-Navelet jets 
using the Brodsky-Lepage-Mackenzie procedure to fix the renormalisation scale are in very good agreement with the observables measured by CMS [40] (see also Section 1).

Preliminary CMS measurements with leading charged particles and leading jets at small transverse momenta have been shown [41], with a focus on understanding the transition from the perturbative to the non-perturbative region. The charged-particle jets (charged particles) are measured in the pseudorapidity range $|\eta|<1.9(2.4)$ for transverse momenta $p_{\mathrm{T}}>1(0.8) \mathrm{GeV}$. The small $p_{\mathrm{T}}$ jet (particle) cross section integrated over $p_{\mathrm{T}}>p_{\mathrm{Tmin}}$ falls steeply at large transverse momenta and becomes flatter towards low $p_{\text {Tmin }}$, showing a significant deviation from the pure perturbative prediction (Fig. 16). The behaviour of the measured cross sections indicates also the importance of saturation effects of parton densities already in the $p_{\mathrm{T}}$ range of a few $\mathrm{GeV}$ in $p p$ collisions at the LHC. The measurements are in general not well described by the Monte Carlo event generators, the shape of the measured $p_{\mathrm{T}}$ distribution is best reproduced by the cosmic-ray model EPOS.
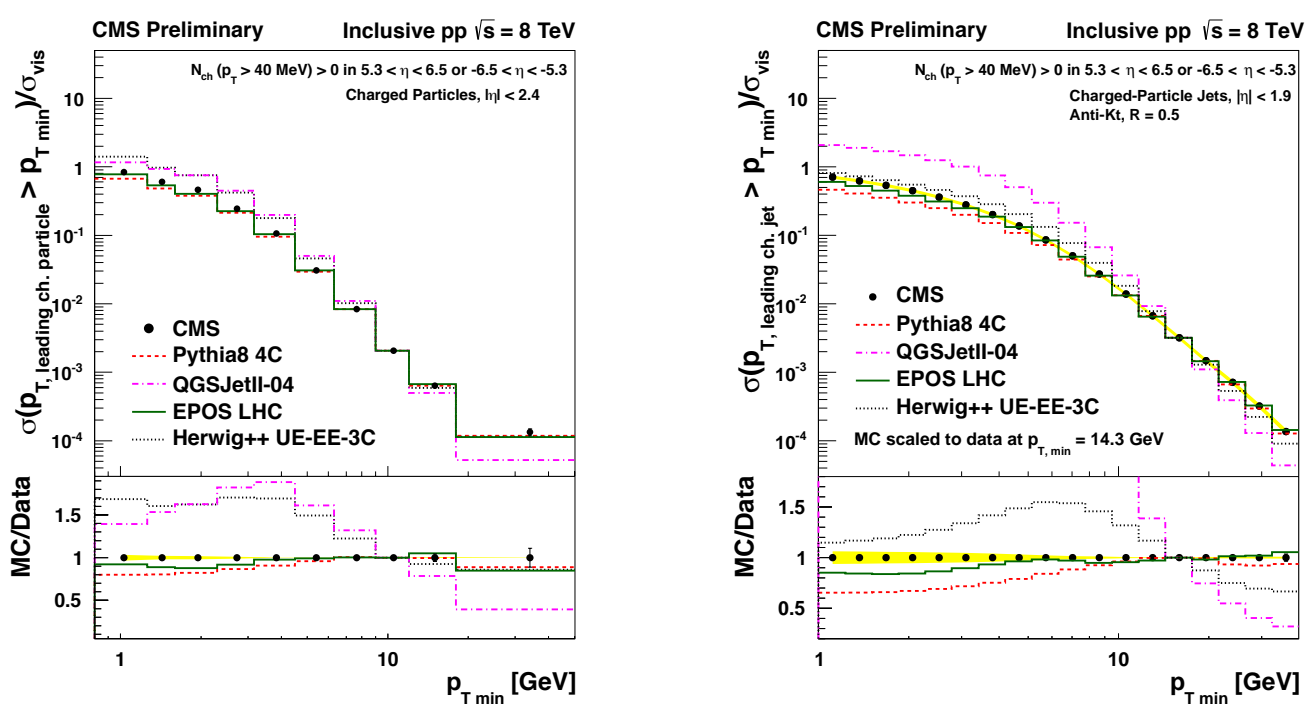

Figure 16: Normalised integrated $p_{\mathrm{T}}$ distribution of the leading charged particle (left) and of the leading jet (right). The data are compared with the predictions of various Monte Carlo generators.

Measurements of inclusive Drell-Yan (DY) production and in association with jets have been reviewd by CMS [42], with the aim to investigate soft gluon resummation. Cross sections as a function of the DY muon pair transverse momentum are measured differentially for various dimuon mass ranges within $30-1500 \mathrm{GeV}$. The cross section for DY production in association with one or two jets with $p_{\mathrm{T}}^{\text {jet }}>30 \mathrm{GeV}$ in the pseudorapidity range $\left|\eta_{\text {jet }}\right|<4.5$ is also studied.

The rise at small $p_{\mathrm{T}}$ of the di-muon $p_{\mathrm{T}}$ distribution for inclusive DY lepton pair production (Fig. 17, left) is a measure of soft gluon resummation. With the DY+1 jet selection the maximum of the $p_{\mathrm{T}}$ distribution is shifted to larger values (Fig. 17, right) and the range of soft gluon resummation is enlarged, thus allowing an observation of perturbative jet resummation. The general behaviour of the distributions is reproduced by the MADGRAPH Monte Carlo generator based on multiparton matrix elements and parton showers. Soft gluon resummation is reasonably well described by initial state parton showers. 

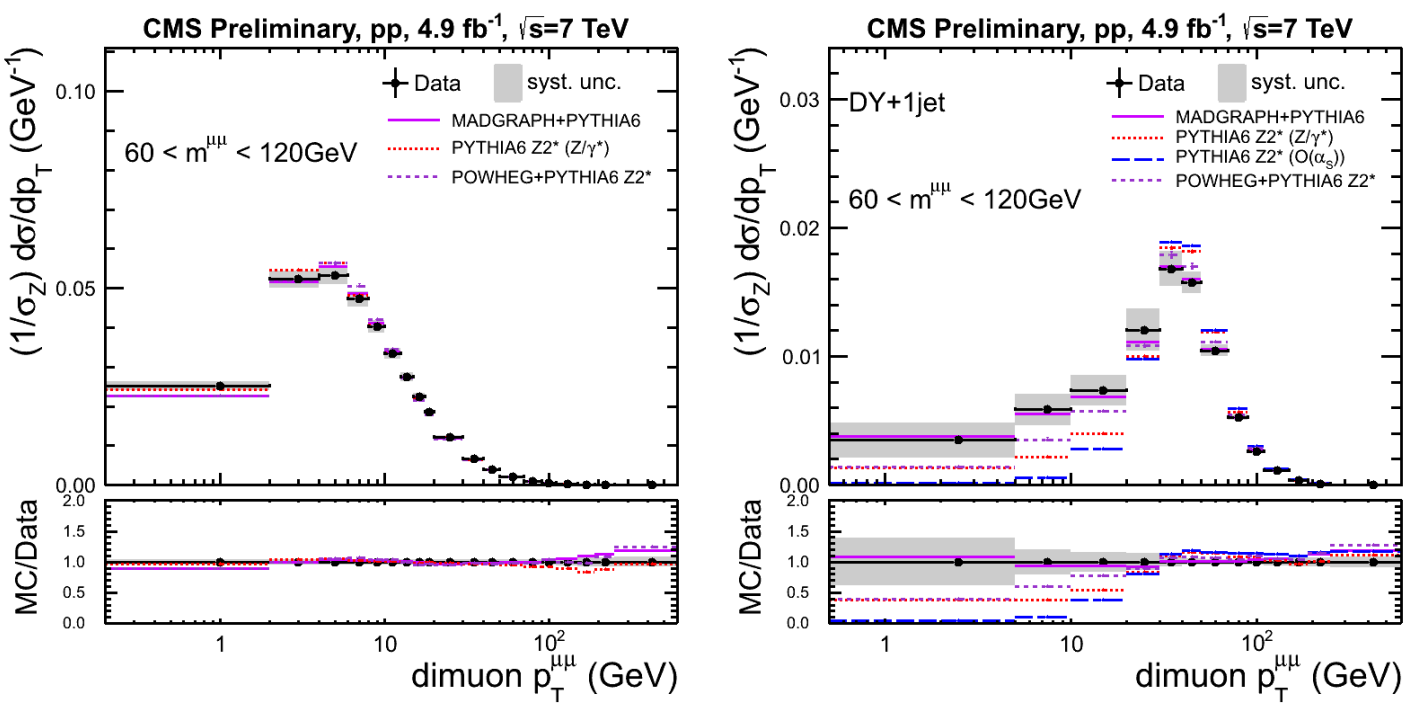

Figure 17: Drell-Yan di-muon transverse momentum distribution normalised to the $Z$ resonance region $(60-120) \mathrm{GeV}$ for inclusive DY (left) and DY+1jet (right) selections.

\subsection{Future Projects}

Two future LHC projects for diffractive physics at high luminosities - and hence high pileup levels - were presented. At Interaction Point 5 the CMS-TOTEM Precision Proton Spectrometer, CT-PPS [43], is based on an upgrade of the TOTEM Roman Pot system that after the Long Shutdown 1 will consist of 26 Roman Pots, partly dedicated to TOTEM's standalone programme with special beam optics, and partly optimised for operation with high-intensity beams in standard LHC fills. To resolve event pileup via longitudinal vertex reconstruction, time-of-flight detectors are developed for installation in Roman Pots.

At Interaction Point 1, the ATLAS Forward Proton project, AFP [44], also aims at an extended Roman Pot system with time-of-flight detectors, based on similar technologies as the counterparts at IP5.

\section{References}

[1] A. Besse, "Leptoproduction of vector meson from the small $x$ to the valence region", these proceedings.

[2] G. Beuf, "Gluon saturation beyond (naive) leading logs", these proceedings.

[3] T. Altinoluk, N. Armesto, G. Beuf, M. Martinez and C. A. Salgado, "Next-to-eikonal corrections in the CGC: gluon production and spin asymmetries in pA collisions," arXiv:1404.2219 [hep-ph].

[4] A. Kovner, M. Lublinsky and Y. Mulian, Phys. Rev. D 89, 061704 (2014).

[5] G. Beuf, Phys. Rev. D 89, 074039 (2014).

[6] G. Chachamis, M. Deak and G. Rodrigo, JHEP 1312, 066 (2013).

[7] K. Kutak and P. Surówka, Phys. Rev. D 89, 026007 (2014).

[8] M. S. Costa, M. Djurić and N. Evans, JHEP 1309, 084 (2013).

[9] A. H. Rezaeian, "b-CGC vs IP-Sat and combined HERA data," these proceedings. 
[10] A. H. Rezaeian and I. Schmidt, Phys. Rev. D 88, 074016 (2013).

[11] A. Luszczak and H. Kowalski, Phys. Rev. D 89, 074051 (2014).

[12] T. Lappi and H. Mantysaari, Phys. Rev. D 88, 114020 (2013).

[13] T. Lappi and H. Mantysaari, Phys. Rev. C 87, no. 3, 032201 (2013).

[14] B. Ducloué, L. Szymanowski and S. Wallon, PoS DIS 2013, 309 (2013).

[15] B. Ducloué, L. Szymanowski and S. Wallon, Phys. Rev. Lett. 112, 082003 (2014).

[16] A. van Hameren, R. Maciula and A. Szczurek, "Single-parton scattering versus double-parton scattering in the production of two $c \bar{c}$ pairs and charmed meson correlations at the LHC," arXiv:1402.6972 [hep-ph].

[17] A. van Hameren, P. Kotko, K. Kutak, C. Marquet and S. Sapeta, Phys. Rev. D 89, 094014 (2014).

[18] A. Cisek, W. Schäfer and A. Szczurek, "Exclusive photoproduction of charmonia in $\gamma p \rightarrow V p$ and $p p \rightarrow p V p$ reactions within $k_{t}$-factorization approach,” arXiv:1405.2253 [hep-ph].

[19] L. Motyka, "On multiple gluon exchange in $J / \psi$ hadroproduction", these proceedings.

[20] A. Szczurek, "Higgs production within $k_{\perp}$-factorization with unintegrated gluon distribution functions", these proceedings.

[21] W. Schaefer, "Central $\mu^{+} \mu$ production via photon-photon fusion in proton-proton collisions with proton dissociation", these proceedings.

[22] J. Malka, "A Review of Diffraction at HERA", these proceedings.

[23] R. Zlebcik, "Diffractive Dijet Production with Leading Proton in ep Collisions at HERA", these proceedings.

[24] M. Guzik, "Exclusive Dijet Production in Diffractive Deep Inelastic Scattering at HERA", these proceedings.

[25] S. Levonian, "Exclusive Photoproduction of $\rho$ Meson with Leading Neutron at HERA", these proceedings.

[26] M. Ruspa, "Diffractive and Exclusive Measurements at CMS", these proceedings.

[27] M. Deile, "Elastic and Diffractive Proton-Proton Scattering Measurements by TOTEM at the LHC", these proceedings.

[28] K. Goulianos, "Predictions of Diffractive and Total Cross Sections at LHC Confirmed by Measurements", these proceedings.

[29] V. Khoze, "Central Exclusive Production in hadron collisions", these proceedings.

[30] P. Lebiedowicz, "Applications of the tensor pomeron model to exclusive central diffractive meson production", these proceedings.

[31] J. Turnau, "Measurement of the Central Exclusive Production of pion pairs using tagged forward protons at the STAR detector at RHIC", these proceedings.

[32] T. Akesson et al., Nucl. Phys. B264 (1986) 154-184.

[33] T. Szumlak, "Exclusive $J / \Psi$ and $\Psi(2 S)$ vector meson production", these proceedings.

[34] O. Villalobos Baillie, "ALICE Results on Ultra-Peripheral Production”, these proceedings. 
[35] L. De Nooij, "Phi meson production measurement with ATLAS", these proceedings.

[36] M. Adinolfi, "Quarkonia production at LHCb", these proceedings.

[37] M. Adinolfi, "Study of Jpsi production and cold nuclear matter effects in $\mathrm{pPb}$ collisions", these proceedings.

[38] G. Safronov, "Exclusive Photoproduction of Beyond-DGLAP Searches with Mueller-Navelet jets, and Measurements of Low- $p_{\mathrm{T}}$ and Foreward Jets at CMS", these proceedings.

[39] P. Cipriano, "Forward-Central Jet Correlations in $p p$ Collisions at CMS", these proceedings.

[40] B. Ducloue, "Confronting BFKL Dynamics with Experimental Studies of Mueller-Navelet Jets at the LHC", these proceedings.

[41] A. Knutsson, "Leading track and leading jet cross sections at small transverse momenta", these proceedings.

[42] S. Dooling, "Measurement of Drell-Yan and Associated Jet Cross Section at Low and High Invariant Masses in $p p$ Collisions at $\sqrt{s}=7 \mathrm{TeV}$ ", these proceedings.

[43] V. Avati, "The CMS-TOTEM Upgrade Programme", these proceedings.

[44] R. Staszewski, "AFP - Forward Protons Detectors for ATLAS”, these proceedings. 